\title{
A Universal Mobile Money Transfer Platform
}

\author{
Kelvin Kinyua Mwangi \\ Faculty of Information Technology, \\ Strathmore University \\ Nairobi, Kenya
}

\author{
Bernard Shibwabo Kasamani \\ Lecturer, Faculty of Information Technology \\ Strathmore University \\ Nairobi, Kenya
}

\begin{abstract}
Mobile money transfer is among some of the fastest growing sectors in Information Technology today. With its continued usage, come new challenges that need to be addressed. Among some of those challenges include the need for a mobile money transfer service that allows transfer of money across all mobile service providers, provide a link to a customer's other electronic sources of money and also allow the user to push money to a group of people with a single transaction.
\end{abstract}

Nexcash is proposed as a platform that allows for the transfer of funds across various network service providers efficiently and effectively using a mobile phone. It provides a link between the user's mobile accounts to other electronic sources of money that they have, particularly banks. It also allows batch sending of money. Among some of the functionalities it has include: sending of money to either an individual or a group, withdrawal of money from Nexcash accounts, deposit of money to Nexcash account, transfer of money to user's different accounts.

Nexcash runs on Global System for Mobile Communications (GSM) networks. The user interacts with the application using their mobile phones, to which transaction menus are forwarded by the application depending on some key-word that the user sends to the application. Nexcash brings in four new benefits into the money transfer solutions industries. It provides openness to all mobile service providers; any user from any mobile service provider is able to use the solution. It allows for batch sending of money to a group of persons with a single transaction. It provides link to a user's bank accounts which allows a user to perform transactions in their bank accounts using their mobile phones. It provides standardized transaction charges regardless of the mobile service provider that your SIM card is subscribed to.

\section{Keywords}

Money Transfer, Mobile Money, Universal Money Transfer, Funds Transfer, Electronic Funds.

\section{INTRODUCTION}

Mobile money (m-money) refers to the use of mobile phones to perform financial and banking functions. It can be used to assist the billions of people who have little or no access to traditional financial services. Where the service is available, users can securely receive funds, pay bills, make bank transactions, transfer funds, and purchase goods and services. Half the households in the world do not have access to financial services. The poor often must rely on informal financial services that may be more costly and less reliable. Low levels of financial inclusion represent an obstacle to economic development. Consequently, financial inclusion has become an important topic in the development agenda.

Mobile money is a sustainable, scalable approach to providing convenient and affordable financial services to the unbanked. More than one billion customers in developing markets have access to a mobile phone, but do not have a formal bank account. Having access to financial services, and somewhere safe to save, greatly improves the lives of the poor. It is true that, historically, financial institutions have been unable to reach low-income customers adequately, especially in remote areas, given the cost of bricks-and-mortar branches. Through mobile money, customers can perform financial transactions [1].

Mobile phone-based money transfer and banking solutions have been recognized as the avenue to take banking services to people outside the formal financial industry. The services have helped reduce the cost of access to financial services for 2.3 billion people in the world who live on less than $\$ 2$ per day and cannot afford formal financial services. Apart from various existing mobile banking solutions in the east African region like M-pesa, Zap and yuCash, there are about 120 other pilot mobile banking services around the world. Mobile banking services have various benefits to the population, including increased productivity and capital flows, helping to manage cash flow as well as enhancing management of erratic incomes [2].

Over the recent past, mobile money transfer has been on the increase with new solutions coming up every day. People, especially Kenyans have embraced the convenience that mobile money brings with it. They have thus opened mobile money accounts with the different mobile service providers including Safaricom, Airtel, $\mathrm{Yu}$ and Orange. These solutions have provided great convenience for Kenyans but still some gaps remain to be filled up. Among one of the major limits of all these solutions is that they tie the subscriber to a certain mobile service provider. This is inconveniencing to most Kenyans since there are several mobile service providers already operating in the country thus a person should be provided with the capability of sending money to whichever network that they wish without limits. Also being tied down to a single mobile service provider means you only have access to your money only in the area that that mobile service provider operates thus convenience is reduced further. The mobile money transfer service should be such that you can be able to carry out transactions via the phone in any place that has mobile network coverage regardless of the mobile service provider operating there [3].

These different solutions from the mobile network service providers also have brought about a major high transaction charge issue with them charging extra high charges for a user to send money to other network [3]. This has further disadvantaged the user by him opting not to send money to a person who is subscribed to a different mobile network service provider because of the high tariff charges.

\section{LITERATURE REVIEW}

\subsection{Mobile Technology Usage in the World}

The increase in the number of mobile cell phones in the world has been impressive. The graph below shows subscriber growth between 2005 and early 2013, according to ITU 
figures. The 6.8 billion subscribers are approaching the 7.1 billion world population. The average world penetration stands at 96.2\%, according to the ITU [4]. Fig 1 and Fig 2 present the mobile phone penetration statistics.

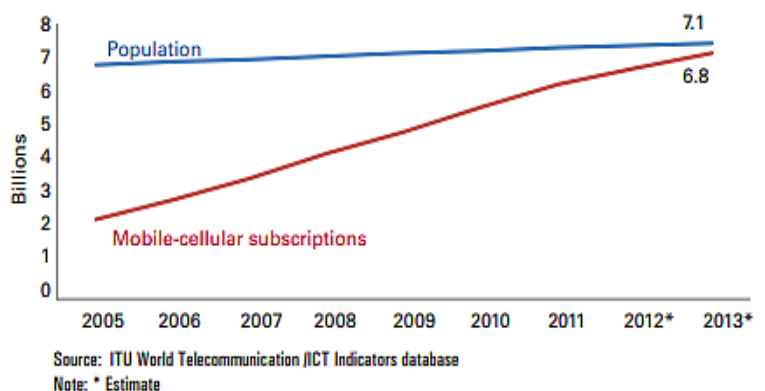

Fig 1: Cell Phone Users in the World [4]

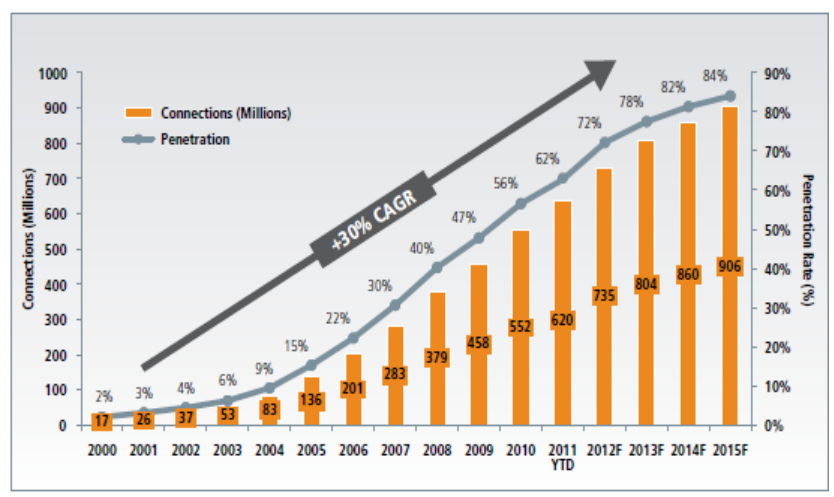

Fig 2: Mobile Phone Penetration in Africa [5]

\subsection{Mobile Money Transfer Solutions}

Among the first mobile network operators in the world to offer branchless banking were Globe Telecom and SMART in the Philippines [6]. In Kenya, a remarkably successful country in mobile funds transaction, four mobile operators (Safaricom, Airtel Kenya, Essar and Telkom Kenya) dominate the market, each offering an independent service to retain customers [7].

The mobile-banking sector has created thousands of jobs through the hiring of agents and brought millions of previously "unbanked" population to the formal banking sector. Populations in rural and remote areas like the North East of Kenya have limited access to banking services due to insufficient branch networks in their locations. Mobile financial services offer efficiency and are time and cost saving for people who have to travel long distances to access a physical bank branch. The introduction of new platforms has the potential to extend the benefits of mobile banking to a wider population [6].

Among all these mobile money transfer solutions limitations still exist. Developers have the opportunity to develop software solutions that could fill the gap where mobile payment solutions have failed to bridge the gap in order to encourage more businesses to deploy the use of mobile money accounts [8]. Among some of the areas for improvements include businesses could avoid payment to wrong numbers using a system that could support incorporating cheque digits when making a payment through mobile money, a follow-up system for paper receipts for clients when they make a payment using mobile money, more applications that enable easy sweeping of funds between mobile money and bank accounts, integrated pay and receive functionality that could reduce the dispute resolution process in the cases of reversal of payments [8].

Another issue that arises from all these solutions is high cost of transactions by the solution providers. These solutions are also provided by specific mobile network service providers thus subscribers to these services are limited to these mobile service providers.

A solution is therefore needed that is first and foremost open to any mobile network service provider so that a user can transact regardless of the mobile service provider they are subscribed to. Also a link to the bank is an essential feature that would be required in that solution so that it allows for convenience to all people, those who already have bank accounts and also those ones that do not have. Finally the solution should be relevant to businesses, which usually carry out bulk transactions thus it should provide a feature for batch processes.

\section{METHOD}

\subsection{System Development Methodology}

This research adopted an Object-oriented System Development methodology. An Object-oriented software design is a design strategy where system designers think in terms of 'things' instead of operations or functions.

Object-oriented development methodology ensures that the system being developed is refined and transformed through analysis, design, code and test phases. Details and modifications are added in successive iterations (changes and improvements are introduced as needed) and incremental releases of software modules are delivered [9].

Object-oriented software development life cycle is an iterative process that has five key phases. The phases of this methodology and which were followed are:

i. Requirements analysis: - This phase is critical to the success of the project. Expectations need to be fleshed out in great detail and documented. This is an iterative process with much communication taking place between stakeholders, end users and the project team [10]. The key stakeholders and users included the University's Head of Security as well as the Security Personnel manning the entry points of the University, the Researcher interacted with them so as to fully understand their day to day processes as well as to collect/gather the desired system features.

ii. Design: - During this phase, the technical design requirements are prepared. The User requirements are used to define how the application will be written; the technical requirements are specified to detail for instance; database to be used, features and functionalities, security processes and hardware and system requirements [11].

iii. Code: - At this stage, the design is translated into a machine-readable form. Programming tools like compilers, interpreters, debuggers are used to generate the code. Different high level programming languages like, C, C++, Java, PHP, HTML are used for coding.

iv. Test: - This stage occurs after the application has been developed; different types of testing will be performed including performance and integration testing. User acceptance testing is the last part of testing and is performed by the end users to ensure 
the system meets their expectations. At this point, defects may be found and more work may be required in the analysis, design or coding [11].

v. Maintenance: - This phase confirms the software passed the user acceptance stage and now is operational. If required, the users are trained on, or aided with the documentation on how to operate the software and how to keep the software operational. The software is maintained timely by updating the code according to the changes taking place in user end environment or technology. This phase may face challenges from hidden bugs and real-world unidentified problems.

\section{SYSTEM DESIGN AND ARCHITECTURE}

\subsection{Requirement Analysis}

A requirement is any function, constraint, or property that the system must provide, meet, or satisfy in order to fulfill its purpose. The goal of analysis is to produce essential requirements.

The user requirements are as follows:

i. User should have a GSM enabled mobile phone.

ii. User should have an account in order to use the system.

iii. User should have a SIM card that is registered to a specific mobile service provider.

iv. User should be conversant with the English language.

v. User inputs the necessary details in order to perform a transaction

vi. User should be conversant with the different transaction term such as withdrawal, deposit, revert, transfer etc.

The System Requirements are as follows:

i. The system provides users with menu that they use to do their different transactions.

ii. The system provides specific menu in response to a user with respect to user's input.

iii. The system keeps the database in sync with the transactions that are carried out by users on the interface.

iv. The system should track down all users' transactions so as to keep their accounts in harmony with the transactions.

The Developer Requirements are as follows:

i. Developer should be conversant with unstructured supplementary service data (USSD) and how it works.

ii. Developer should be conversant with SMS threading and how it is implemented.

iii. Developer should have knowledge on OzekiSMS server.

iv. Developer should be comfortable using visual studio or any other text editor that can support asynchronous server pages (asp).

\subsection{System Architecture}

Nexcash runs on global system for mobile communication (GSM). The following are the main components in the system:

\section{i. User Interface}

The users accessed the system using their mobile phone and doing their different transactions. They were be submitting their transactions via SMS, which were routed to the Ozeki SMS gateway using their mobile network service provider's network.

\section{ii. SMS gateway}

Ozeki Server was used as the SMS gateway. It was picking the user input from the user interface and committing it to the application and was also picking information from the application and submitting the menu to the users.

\section{iii. Application}

The user input was being routed to the application by Ozeki SMS gateway. In this application was where all the processing of the user transactions took place. Asynchronous server pages (asp) were used to provide the code for the different functionalities in the application. Microsoft SQL Server was the database tool and it was keeping all the tables that were in the application.

Fig 3 presents the architecture of the system.

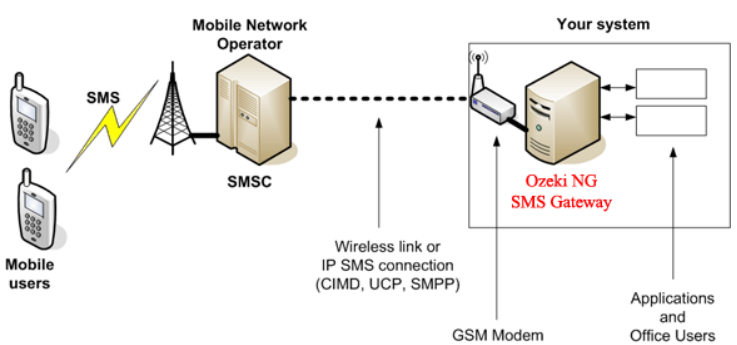

Fig 3: System Architecture - SMS [12]

\subsection{Use Case Diagram}

A use case diagram at its simplest is a representation of a user's interaction with the system and depicting the specifications of a use case. A use case diagram can portray the different types of users of a system and the case and will often be accompanied by other types of diagrams as well. Fig 4 shows a use case diagram describing the actors and the activities involved in the new vehicle registration process.

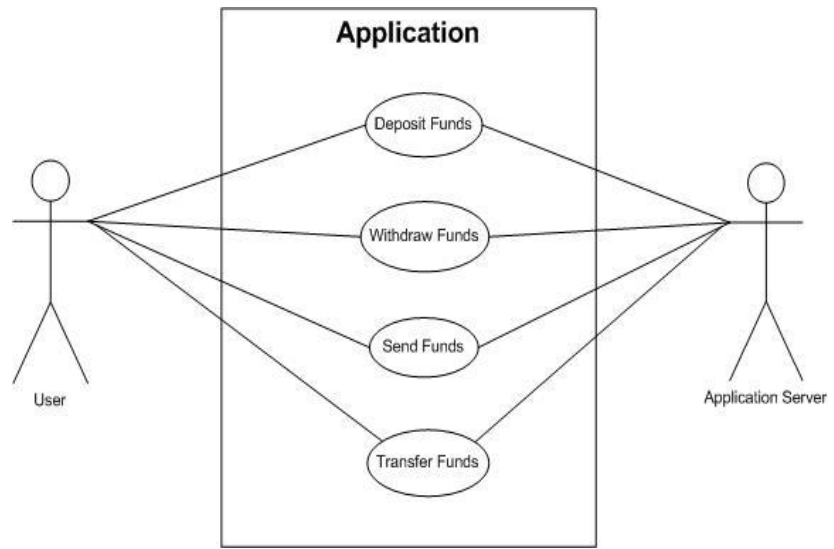

Fig 4: Use Case Diagram for the Universal Mobile Transfer Platform 


\subsection{Activity Diagram Descriptions}

The following are descriptions for the activity diagram:

\section{i. User Chooses 'Send'}

A user sends a keyword in order to get the application menus. The application responds to the user by sending them their respective menus. The user chooses the option of sending after which the application responds by asking the user for their pin. If the user does not provide the correct pin, the application asks again and if the user fails to provide the correct pin after a certain number of times the application drops the transaction and exits. After user provides the correct pin, the application asks the user for the amount that he wants to send. If the user does not input the correct amount the application asks him for the amount again and if he fails to input the correct amount several times the application quits the transaction and logs off the user. Once they have specified amount correctly user is asked for the recipient they are sending money to. The application verifies that recipient has an account. If they have an account, the application checks that the amount specified can be transacted through checking the recipient and senders balance. It then sends them notifications with details of the transaction using their mobile network service provider's network.

\section{ii. User Chooses 'Bank'}

A user sends the keyword in order to get the application menus. The application responds to the user by sending them their respective menus. The user choses the option of bank after which the application responds by sending the user the bank menus. User choses the option of transferring cash to bank and the application prompts user for their pin. Once the application verifies the pin it asks the user for the bank account that they want to transfer cash to. If the bank account does not exist, the application asks again and logs user off after several failures. If the bank account is valid, the application asks the user for the amount they want to transfer. If the amount is correct and user has enough balance in their account to carry out the transfer, the application carries out the transaction and notifies the user.

\section{iii. User Chooses to Send to Group}

A user sends the keyword in order to get the application menus. The application responds to the user by sending them their respective menus. The user choses the option of group after which the application responds by sending the user the group menus. User choses the option of sending money to group and the application prompts the user for their pin. After verifying user pin is correct application asks the user for the group they want to send money to. After the application confirms the group exists, it checks the user balance to ensure that they have enough balance to send the amount and carries out the transaction. It notifies the user and logs them off.

\subsection{Database Design}

Fig 5 shows the database schema, the different application tables and their relationships. In the diagram there is the users table which is keeps details of the application users (admin, agent and standard user). There is the groups table which keeps the various groups that a certain user creates. A user has many groups hence has a one to many relationship with the group. A group has group members hence the foreign key relationship with the group members table. In summary group members belong to a certain group which belongs to a certain user. There is the accounts table which keeps details of the user accounts. An account belongs to a certain user hence the foreign key relationship shown on the diagram. User has a certain gender as shown by the relation on the diagram. The agent registrations table keeps record of all the user registrations performed on the application. These may be agents or users. An agent is registered by the application administrator while the user is registered by agent.

Account types table keeps record of the different types of accounts the application has and every account falls under a certain account type. Deposits records the different deposits that happen in the application, the account from which the money moves from to the account the money is deposited. It thus has a foreign key relationship with accounts. Also details of the amount, time and code of the deposit is recorded in the transactions table hence they are related.

Transactions table keeps details of the different transactions that go on in the application. A transaction can either be a withdrawal, deposit or transfer. Thus details about a specific transaction are recorded on either the deposits, withdrawals or transfers table respectively.

Transaction types keeps details of the various transaction types occurring in the application. Each transaction belongs to a specific transaction type and each transaction type has a specific transaction charge. Transaction charges stores the various charges available for any transaction. 


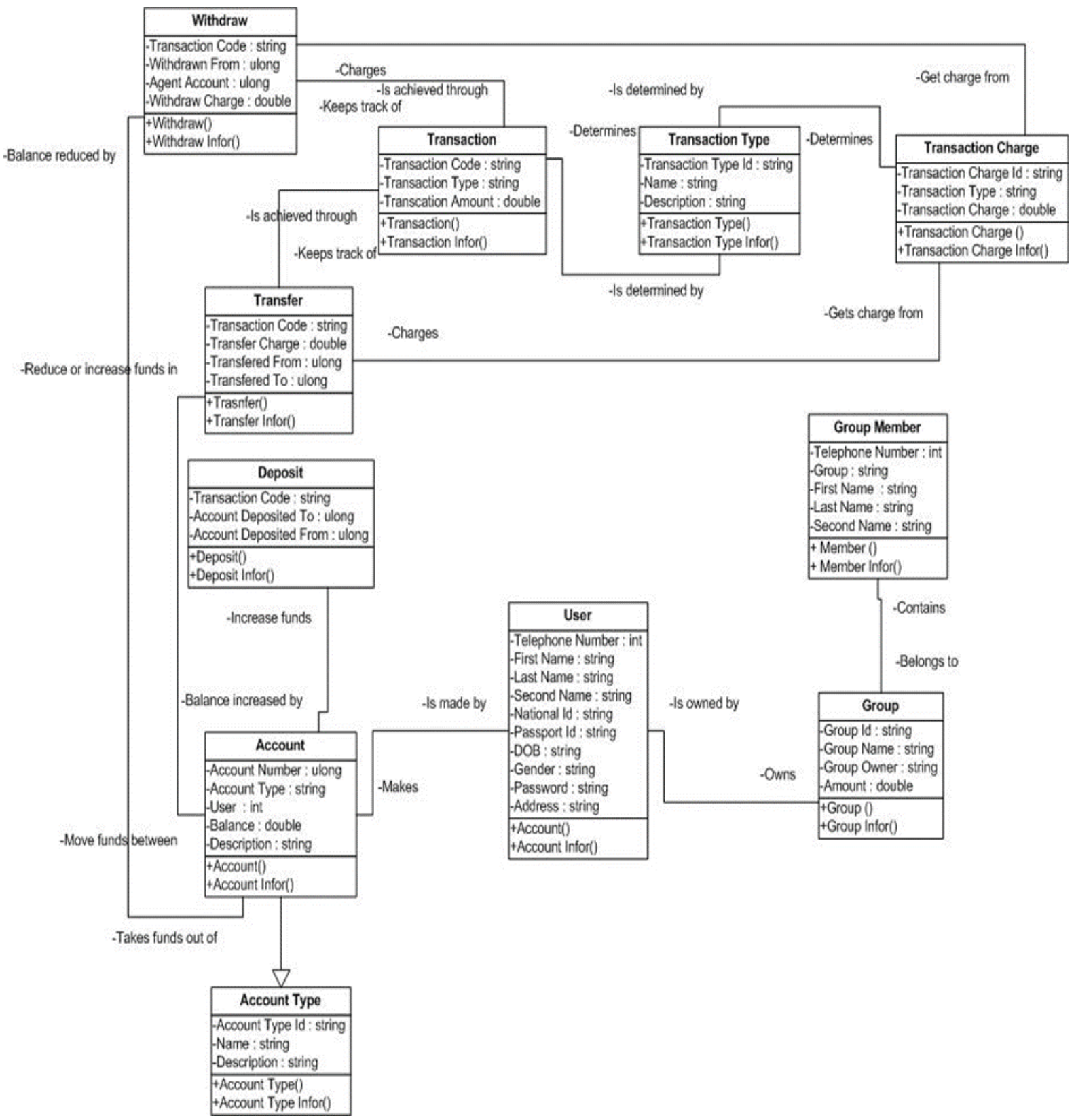

Fig 5: Database Schema for the Universal Mobile Transfer Platform

\section{SYSTEM IMPLEMENTATION AND TESTING}

\subsection{System Development Tools}

This system architecture is split into two main categories namely the client side and the server side. The solution was implemented on a phase basis providing a different functionality in each phase as stipulated in the project plan. The project was implemented using Windows 7 operating system, Microsoft SQL Server Express 2012, Visual Studio 2010 and Ozeki Server SMS gateway. By the time all the phases were complete, all the functionalities required in the solution were met.

\subsection{Application Frontend}

Nexcash application front end runs on Ozeki-NG SMS Gateway. It runs as an ASP application which has a script in which all the different functionalities are achieved. The script is where all the user menus are defined and also where all the code that processes user requests is contained. The front end application has two interfaces, the nexcash agent interface and the nexcash standard user interface. Both of these users have different uses in the application hence are served with different menus by the application. The interaction between the application and the user is via SMS menus. The user sends SMS to the application and the application receives and processes incoming user SMS. The application is also connected to the nexcash database such that whatever transactions happen in the front end they are committed to the database ensuring the whole nexcash application is in sync. Via the front end, the agent is able to register a nexcash standard user, deposit money to a nexcash standard user account and check details concerning their own account. The standard user is able to send cash to other users, withdraw cash from his account, check his account details, transfer cash to and from a bank account and be able to send cash to a group of users.

Fig 6 shows the application main menus, the agent main menu 
and the standard user main menu both of which show the main application functionality of the nexcash agent and the user.

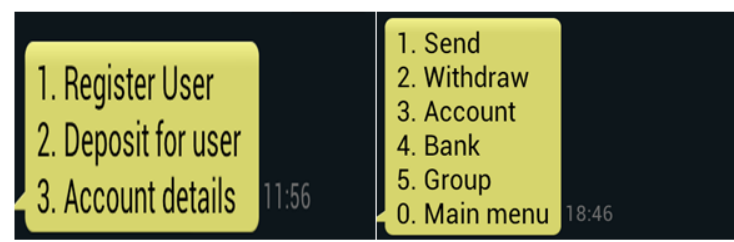

Fig 6: Nexcash Main Menu

Fig 7 shows the agent registration process. The agent choses the register user option from the main menu. The application replies with a message directing the agent on how to register a user. The agent inputs the user details plus his pin. The pin is used to verify the identity of the agent. Once the agent submits the information the systems verifies that agent exists and validates the user details. If the details are correct a nexcash standard user account is opened for the user and a notification is sent to the user.
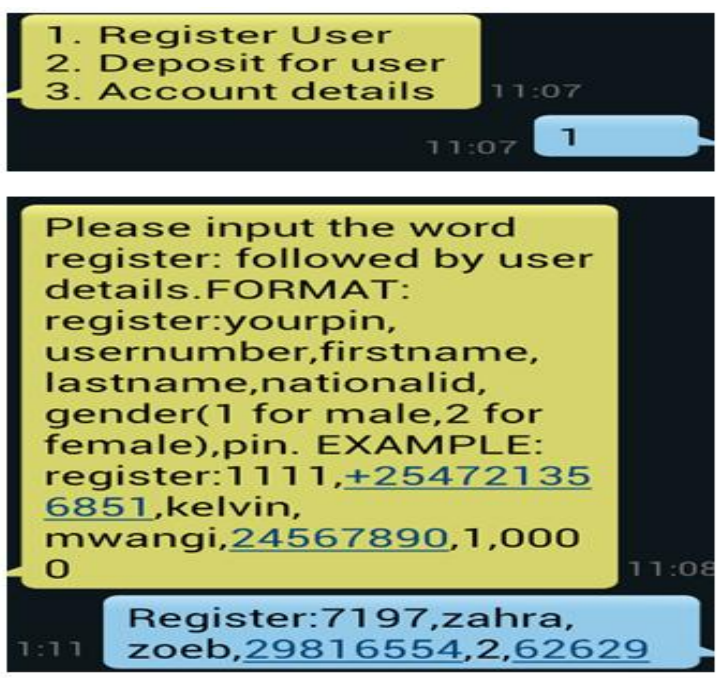

Fig 7: Agent User Registration

Fig 8 shows the deposit procedure. The agent chooses the option to deposit for user and the application replies to him with the directions on how to deposit for a user. The agent fills in the details and sends the reply back to the application. The application verifies that he details are correct after which it proceeds to carry out the transaction. It checks that the agent has enough balance to deposit for the user and that the user account can be able to receive the amount deposited. The application then sends a notification to the user informing them of the deposit. Fig 9, Fig 10 and Fig 11 present additional operations that can be achieved.

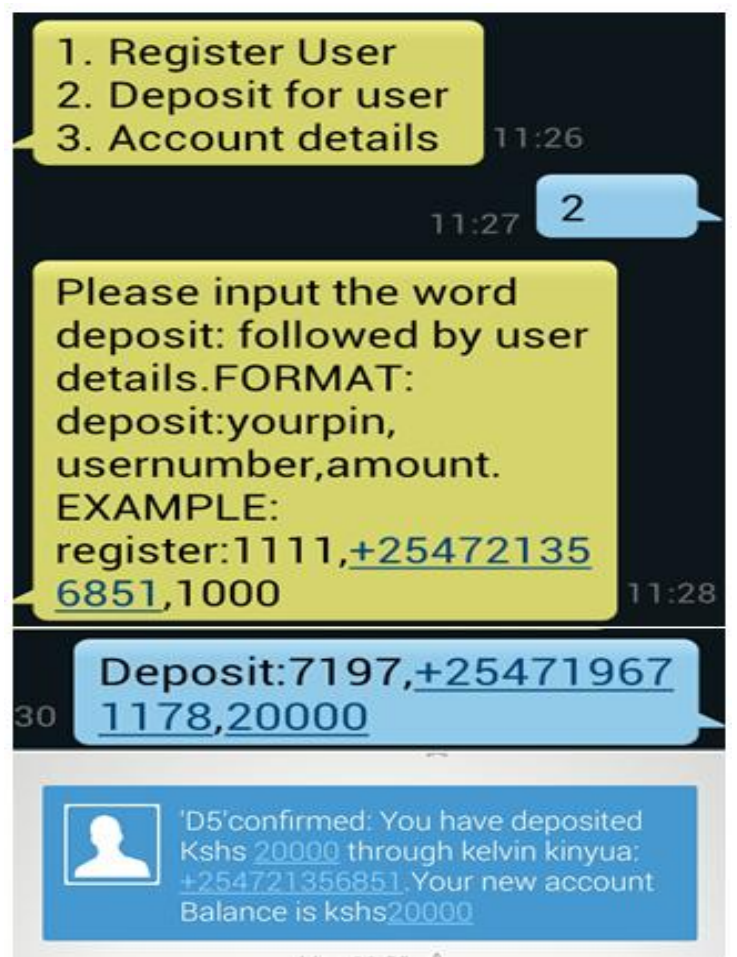

Fig 8: Agent Deposit Option

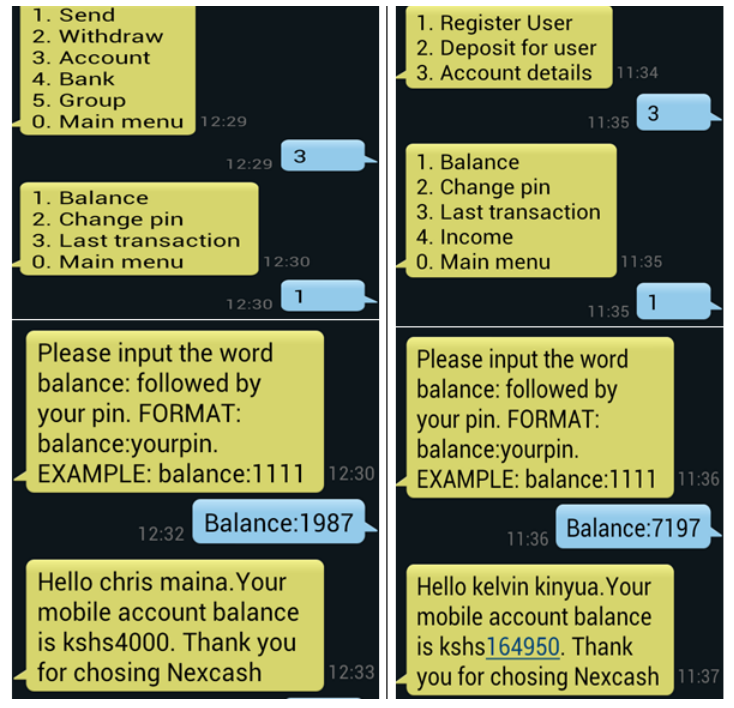

Fig 9: Balance Enquiry 

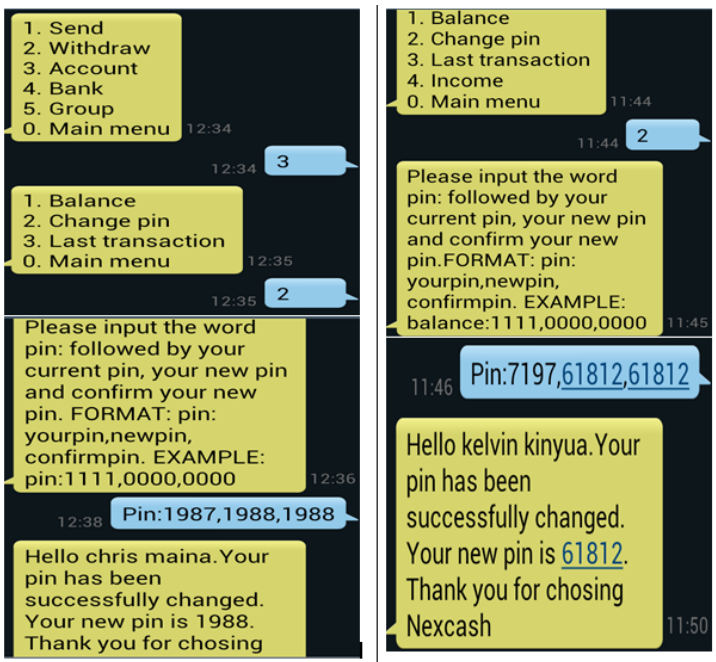

Fig 10: Change PIN option

Fig 11 shows how an agent gets to inquire about the income he has earned through his transactions. The agent choses the option income in his account menu, the application asks the agent for his pin. After verification, the application replies back to the user with details about his account balance.

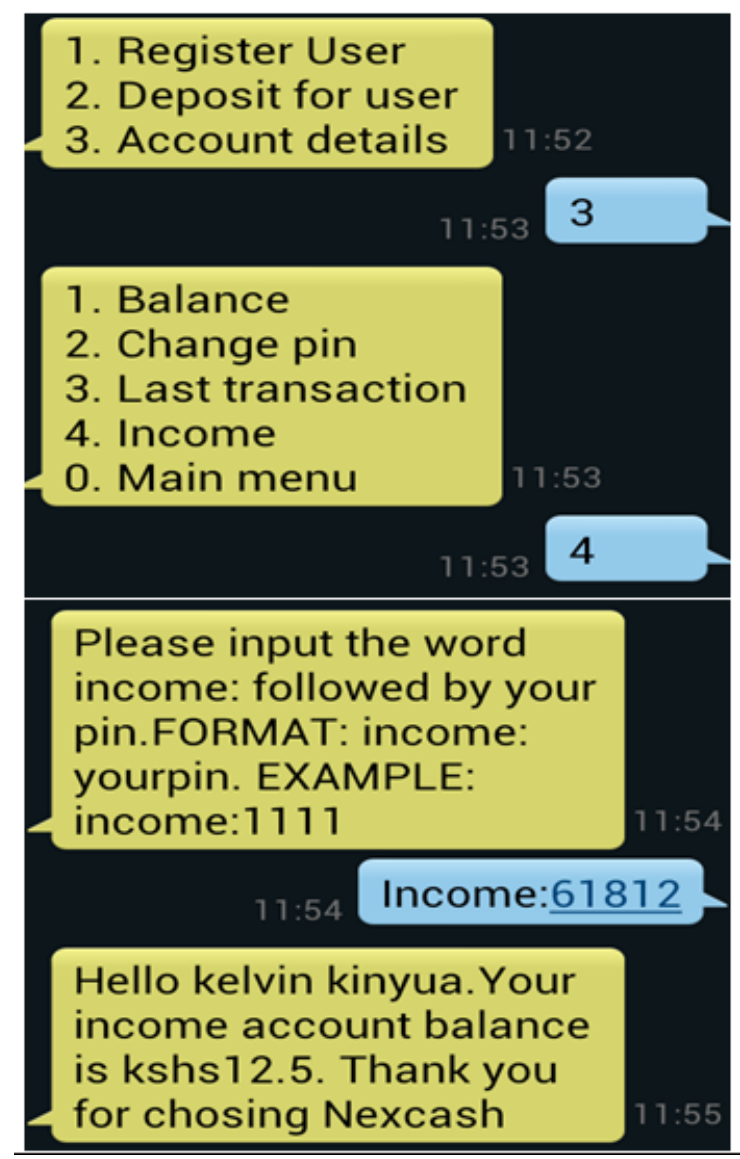

Fig 11: Agent Income Inquiry

Fig 12 shows the process of group adding by user and also the process of sending funds to a certain group.

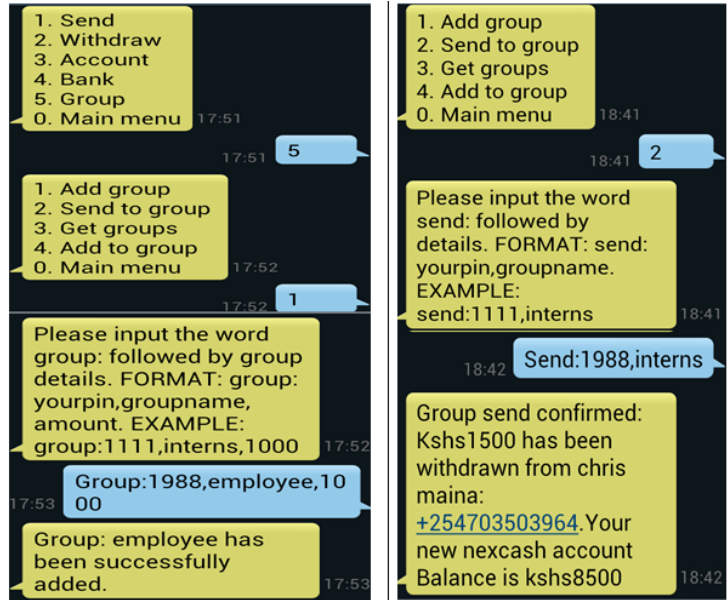

Fig 12: Group Add and Send Process

\subsection{Application Backend}

The application back end is implemented using visual studio 2010. The programming language used is C\# web MVC 4 application. The back end is for monitoring, reporting and administration of the application. Fig 13 shows a section of the backend functions which include a reports and analytics page.

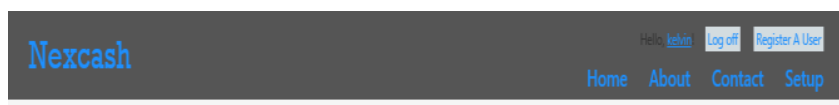

\section{Nexcash Setup and reports}

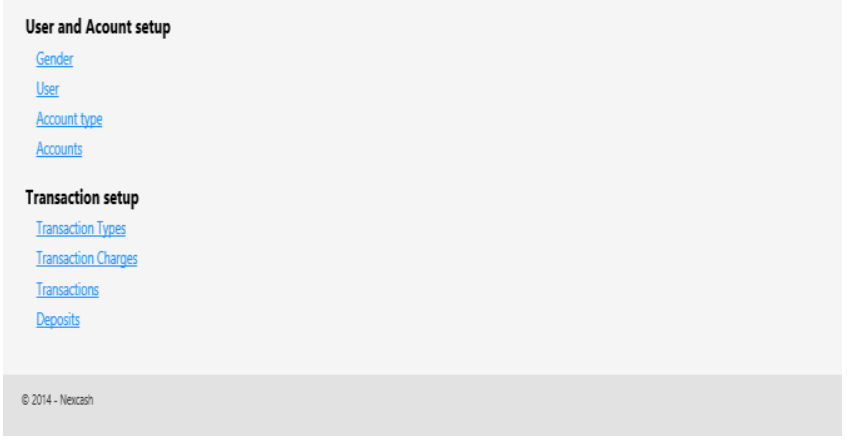

Fig 13: Backend Setup Page

\section{CONCLUSION}

Nexcash has met all the objectives set forth in its inception including: sending of money to either an individual or a group, withdrawal of money from user's different linked accounts, deposit of money to user account, transfer of money to user's different accounts and it is open to all mobile network service providers. Each of these goals was met by providing functionality for each in the application thus the application grew incrementally meeting all the goals. Nexcash is a fully fledged money transfer solution that promises to bring a difference in the mobile money transfer industry.

The implementation methodology was a great one that allowed the delivering of the solution in phases allowing the development of the project from the database, to the back end and finally the front end application. The tools used met all the requirements set forth thus allowing the delivering of the solution on time.

In future, Nexcash is essentially supposed to also run on 
USSD. Additionally, at the time of developing the solution, Ozeki only provided the SMS functionality, thus for future development other tools, for example Kannel, the powerful open source WAP and SMS gateway which provides for USSD functionality may be used. For the solution to be rolled out to the market, agents will be set up as well and associated customer care services are expected to open up opportunities for employment.

\section{ACKNOWLEDGEMENTS}

Thank you Strathmore University for the support in this study, especially thanks to the Faculty of Information Technology.

\section{REFERENCES}

[1] GSMA. 2013. Mobile World Congress 2013. Retrieved July 17, 2013, from GSMA Web site: http://www.GSMA.com

[2] Deloitte, 2014. Value of connectivity Economic and social benefits of expanding internet access.

[3] Kinyoda, M. 2012. Battle of the Mobile Money Transfer Service. Retrieved July 12, 2013, from Pesa Talk Web site: http://www.pesatalk.com

[4] Internet World Stats. 2013. Mobile Internet - Mobile Phones. Retrieved July 18, 2013, from Internet World Stats Web Site: http://www.internetworldstats.com

[5] T.Phillips, P. L. (2011). Driving Economic and Social
Development through Mobile Services. London: GSM Association.

[6] FSD Kenya. 2009. Mobile payments in Kenya. Nairobi: FSD Kenya.

[7] Mulupi, D. 2011. Kenya Taking Mobile Money a Step Further. Retrieved july wednesday, 2013, from AudiencScapes web site : http:// AudiencScapes.com

[8] Anne. 2012. Why Doesn't Every Business In Kenya Have A Mobile Money Account? Retrieved july 12, 2013, from ihub website: http:// ihub.co.ke

[9] Leszek A. M. 2001. Requirements Analysis and Systems Design

[10] Justin 2013. What is the Software Development Life Cycle. Retrieved from https://airbrake.io/blog/insight/what-is-the-softwaredevelopment-life-cycle

[11] March, D. 2002. Requirements Definition. Retrieved from http://www4.desales.edu/ dlm1/it532/class01/reqdef.htm

[12] Ozeki Informatics ltd. (2000-2013). index1737.html. Retrieved july 10, 2013, from Ozeki informatics limited web site: http://www.ozekisms.com 